\title{
Crosstalk Mitigation for LTE-over-Copper in Downlink Direction
}

\author{
Eduardo Medeiros, Yezi Huang, Thomas Magesacher, Stefan Höst, \\ Per-Erik Eriksson, Chenguang Lu, Per Ödling and Per Ola Börjesson
}

\begin{abstract}
Radio-over-copper is a niche idea that has potential to become a cornerstone in the deployment of dense $5 \mathrm{G}$ networks. We address one of the remaining hurdles and present an architecture for transparent crosstalk mitigation in LTE-over-copper systems. By taking advantage of reference symbols present in the downlink LTE signals we propose two methods for estimating the copper channel. System performance is evaluated using channel measurements and error vector magnitude calculations with promising results.
\end{abstract}

\section{INTRODUCTION}

$\mathbf{H}$ IGH-capacity residential small cells represent an opportunity for operators to increase coverage and penetration of their mobile broadband services. With this paradigm, operators can deliver higher bitrates where most of the content consumption occurs. Additionally, cell site costs and energy consumption can be significantly reduced.

Residential small cells may become even more attractive with the impending introduction of Long Term Evolution (LTE) over unlicensed spectrum bands. Wireline operators, for example, could complement their product portfolio by reusing the copper plant to offer mobile subscriptions.

Early works such as [1] suggest a centralized radio-overcopper concept as an alternative to uncoordinated femtocell deployment. In [2], transparent remote radio heads (RRHs) and shared baseband processors are used to achieve full coordination between small cells and the macro layer, eliminating the main drawback of femtocells. This system in particular targets the enterprise market and takes advantage of shielded networking cables (category 6 and up) to deal with crosstalk and radio frequency interference (RFI).

In [3], we proposed an architecture for small cell deployment that reutilizes the copper access infrastructure (unshielded category 3 cables), while coexisting with legacy digital subscriber line (DSL) services. The proposed system converts baseband radio signals to an intermediate frequency, adequate to the low-pass copper channel and could be deployed in cabinets collocated, for instance, with VDSL2 modems.

In [4], we analyzed the implications of Third Generation Partnership Project (3GPP) compliance to the reach of an

E. Medeiros, Y. Huang, T. Magesacher, S. Höst, P. Ödling and P. O. Börjesson are with the Department of Electrical and Information Technology, Lund University, Sweden, email: \{firstname.lastname\}@eit.lth.se

P-E. Eriksson and C. Lu are with Ericsson Research, Kista, Sweden, email: \{per-erik.s.eriksson, chenguang.lu\}@ericsson.com

This work was partly supported by the Celtic-Plus project GOLD, the EU H2020 5G-Crosshaul project (grant no. 671598) and the EXAM project of EIT Digital.

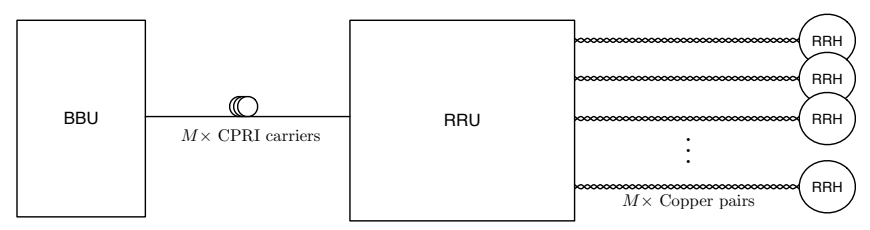

Fig. 1. Centralized Baseband Architecture for an LTE-over-copper system.

LTE-over-copper solution. The analysis indicated that a 3GPP compliant system could be built at a reasonable price point and deployed up to 350 meters away from the cabinet.

While papers [3], [4] have covered bandplanning, rate-reach simulations and filter design, they have not dealt with the main impairment present in copper networks: crosstalk. Solutions for mitigating crosstalk among synchronized transmitters have long been adopted in the DSL community, where these techniques are referred to as vectoring. In brief, vectoring stands for the joint processing of transmit or receive symbols in DSL systems. Works such as [5], [6] proposed efficient algorithms for achieving near-optimal crosstalk cancellation in vectored systems. The International Telecomunication Union (ITU) standardized interfaces for VDSL2 vectoring with the G.993.5 recommendation [7]. Vectoring is also part of the newer G.fast standard [8].

Ideally, the crosstalk problem we address here would be solved by implementing vectoring in the baseband unit (BBU) software, see the left part of Figure 1. However, proposing to change the BBU software to adapt to one particular fronthaul technology cannot be expected to be met with great enthusiasm as it complicates software architecture and maintenance. A solution needs to be implemented further out and kept transparent to the BBU.

The contribution of this letter is threefold: First, we present an architecture for channel estimation and precoding that mitigates the effects of crosstalk on downlink LTE-over-copper systems. Second, we propose two methods for estimating the copper channel using the reference signals already present in LTE signals. Last, we validate the concept using channel measurements and error vector magnitude (EVM) calculations.

\section{Frequency Domain Precoding at RRU}

Consider the centralized baseband system represented in Fig. 1. A single shared BBU processes LTE signals for $M$ independent LTE small cells. The baseband signal samples are transmitted using an appropriate protocol (e.g. CPRI) to 


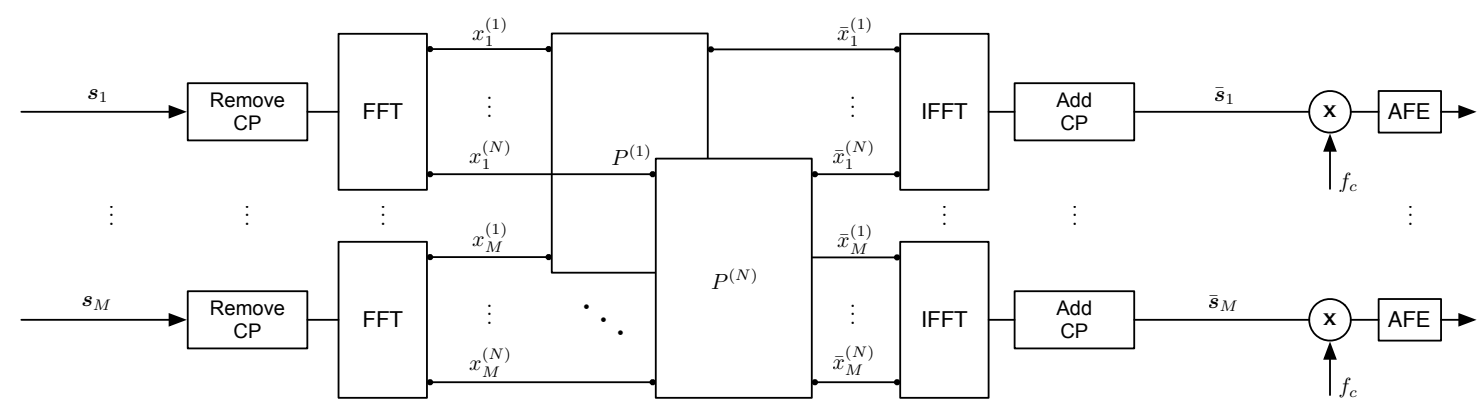

Fig. 2. Proposed remote radio unit architecture. The samples for $M$ independent LTE cells are present at the input, have their cyclic prefixes stripped, are brought to frequency domain and precoded, being transformed back to time domain before modulation and transmission over the twisted pairs.

a remote radio unit (RRU). The radio unit is responsible for up and down-conversion to an intermediate frequency $f_{c}$ suitable for transmission over copper. Each twisted pair is terminated by a remote radio head (RRH), responsible for filtering, conversion to RF frequencies and amplification before sending the LTE signal to an antenna.

Assume that all $M$ independent cells are synchronized, that each cell transmits orthogonal frequency-division multiplexing (OFDM) symbols with $N$ subcarriers and that these cells employ the same duplexing method.

Let $x_{i}^{(k, l)}$ represent the frequency domain contents of the resource element ${ }^{1}$ with OFDM symbol $l$, subcarrier $k$ and transmitter $i$. Dropping the symbol index and focusing the analysis on a single resource element, the following description applies. Let $\boldsymbol{x}^{(k)}=\left[x_{1}^{(k)}, \ldots, x_{i}^{(k)}, \ldots, x_{M}^{(k)}\right]^{\mathrm{T}} \in \mathbb{C}^{M}$ represent the transmitted symbol vector obtained by stacking the output of $M$ synchronized cells at subcarrier $k$. At the output of the copper channel, the received frequency domain symbol vector $\boldsymbol{y}^{(k)} \in \mathbb{C}^{M}$ can be described as

$$
\boldsymbol{y}^{(k)}=\boldsymbol{H}^{(k)} \boldsymbol{x}^{(k)}+\boldsymbol{z}^{(k)},
$$

where $\boldsymbol{H}^{(k)} \in \mathbb{C}^{M \times M}$ is the frequency domain channel matrix for subcarrier $k$ and $\boldsymbol{z}^{(k)} \in \mathbb{C}^{M}$ represents an additive noise vector.

The signal received at RRH $i$ and subcarrier $k$ can then be expressed as

$$
y_{i}^{(k)}=H_{i, i}^{(k)} x_{i}^{(k)}+\sum_{j \neq i} H_{i, j}^{(k)} x_{j}^{(k)}+z_{i}^{(k)} .
$$

where $H_{i, i}^{(k)}$ represents the direct channel gain for pair $i$ and $H_{i, j}^{(k)}, i \neq j$ represents the far-end crosstalk (FEXT) from RRU transmitter $j$ to RRH $i$.

If the off-diagonal elements of the channel matrix $\boldsymbol{H}^{(k)}$ are non-negligible, as is typical for unshielded telephony-grade cables, the crosstalk contribution will significantly distort the LTE signal before it is up-converted and transmitted via the RRH radio frequency (RF) front-end, possibly leading to unacceptable EVM increase.

In this letter we propose to modify the design of the remote radio unit in order to eliminate crosstalk via frequency domain precoding. The proposed arrangement, depicted in Fig. 2, has

\footnotetext{
${ }^{1}$ The smallest time-frequency resource in an LTE grid.
}

the benefit of being transparent to the baseband processor, avoiding the necessity of making the radio access equipment aware of the fronthaul medium.

Let $\boldsymbol{s}_{i}=\left[s_{i}^{(N-L+1)}, \ldots, s_{i}^{(N)}, s_{i}^{(1)}, \ldots, s_{i}^{(N)}\right]^{\mathrm{T}} \in \mathbb{C}^{(L+N)}$ be a vector constituted by $L+N$ samples of the $i^{t h}$ RRU input signal, covering exactly one OFDM symbol, including its cyclic prefix of size $L$. The vector $\tilde{\boldsymbol{x}}_{i}=$ $\left[x_{i}^{(1)}, \ldots, x_{i}^{(k)}, \ldots, x_{i}^{(N)}\right]^{\mathrm{T}} \in \mathbb{C}^{N}$ containing the transmit symbols for every subcarrier used by transmitter $i$ can be obtained by cyclic prefix removal followed by a discrete Fourier transform (DFT). A matrix representation of such operation is given by

$$
\tilde{\boldsymbol{x}}_{i}=\mathcal{F} \boldsymbol{R} \boldsymbol{s}_{i}
$$

where $\boldsymbol{R}$ is the cyclic prefix removal matrix and $\mathcal{F}$ is a DFT matrix of size $N$.

Next, the transmit symbols for each user on subcarrier $k$ can be collected in a vector $\boldsymbol{x}^{(k)}=\left[x_{1}^{(k)}, \ldots, x_{i}^{(k)}, \ldots, x_{M}^{(k)}\right]^{\mathrm{T}}$ and used as input to the precoder. The precoded symbol vector $\overline{\boldsymbol{x}}_{k}$ is obtained via the matrix multiplication $\overline{\boldsymbol{x}}^{(k)}=\boldsymbol{P}^{(k)} \boldsymbol{x}^{(k)}$, where the precoding matrix $\boldsymbol{P}^{(k)} \in \mathbb{C}^{M \times M}$ is chosen appropriately. Good candidate precoder designs are presented in [6], with the diagonalizing precoder shown to be near optimal.

After precoding an inverse DFT is performed on each sequence $\overline{\boldsymbol{x}}_{i}=\left[\bar{x}_{i}^{(1)}, \ldots, \bar{x}_{i}^{(k)}, \ldots, \bar{x}_{i}^{(N)}\right]^{\mathrm{T}} \in \mathbb{C}^{N}$, followed by cyclic prefix insertion. The resulting precoded time domain samples $\bar{s}_{i}$ go through digital to analog conversion and are shifted to the carrier frequency $f_{c}$ before copper transmission.

For good precoder performance it is of fundamental importance that good estimates of the channel matrices $\boldsymbol{H}^{(k)}$ are available. In the next section we describe practical methods for obtaining these estimates.

\section{Channel Estimation Methods}

The LTE physical layer [9] specifies a number of reference signals used in the communication between base stations and UE to achieve different objectives such as cell identification, cell selection and channel estimation.

We propose to utilize LTE's downlink cell-specific reference signals (CRS) to estimate the copper channel. CRS are well suited for the task at hand since they are distributed along the entire bandwidth of the cell and are independent of cell load and scheduling algorithms. 

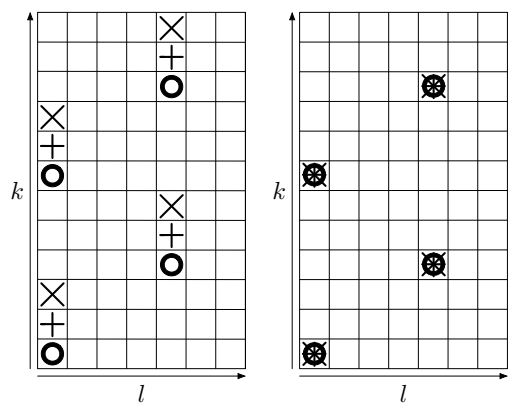

Fig. 3. Depiction of a stack of LTE resource grids for three cells. On the left, the first slot of a resource block is represented, and the cells are assigned the cell identities 0,1 and 2 . On the right, with the proposed cell assignment scheme, one notices that the cell reference symbol positions coincide in time and frequency. The cell identities for the second diagram are 0,6 and 12 .

For each slot in a subframe, CRS are present in the first and fifth symbols. The values of CRS symbols are obtained by QPSK modulating a pseudo-random Gold sequence [9]. By knowing the physical layer cell identity, $N_{I D}^{\text {cell }}$, the slot number within a radio frame $n_{s}$ and the symbol number $l$ one can perfectly reconstruct each of the CRS sequences.

A frequency shift derived from the $N_{I D}^{\text {cell }}$ parameter also controls which subcarriers will be loaded with pilot symbols. This cell-specific frequency shift, defined as $v_{\text {shift }}=$ $N_{I D}^{\text {cell }} \bmod 6$, was introduced to improve pilot performance in environments with high interference.

The effect of different $N_{I D}^{\text {cell }}$ assignments on pilot positioning is exemplified in the left diagram of Fig. 3. The pilot symbols for three different cells are drawn over the same resource grid. Each cell's pilots are represented by the symbols $\circ,+, \times$ and correspond to the choice of cell identity $N_{I D}^{\text {cell }}=0,1,2$ respectively. For this sequential cell identity assignment, the pilot symbols clearly do not overlap.

Taking advantage of the cell-specific frequency shift, we propose that instead of using an arbitrary cell identity assignment, the $N_{I D}^{\text {cell }}$ values should be selected according to the following sequence

$$
\begin{aligned}
& N_{I D}^{\text {cell }}(1) \in\{0,1, \ldots, 503\}, \\
& N_{I D}^{\text {cell }}(i)=\left(N_{I D}^{\text {cell }}(i-1)+6\right) \bmod 504, i \in\{2, \ldots, M\} .
\end{aligned}
$$

When the $N_{I D}^{\text {cell }}$ parameters are set according to Eq. 4, pilots for each cell are assigned to the same resource elements. This is represented in the right diagram of Fig. 3. Based on this pilot alignment we propose two channel estimation architectures, that differ in the amount of feedback necessary between RRH and RRU.

\section{A. Feedback-based estimator}

For the following discussion, it is assumed that there exists a control channel between the RRU and each RRH. It is also assumed that each RRH is capable of synchronizing to its LTE signal, extract the received CRS symbols and feed them back to the RRU. We also assume that the copper channel is constant over many symbols, changing slowly due, for example, to temperature variations [10]. The formulations presented next are valid for subcarriers in which pilot symbols are transmitted.

Let $W, W \geq M$, represent the number of pilot symbols in an observation window. Let $r$ denote the pilot symbol number within an observation window. Define $\boldsymbol{\eta} \in \mathbb{C}^{W M}$ and $\boldsymbol{X} \in$ $\mathbb{C}^{W M \times M^{2}}$ as

$$
\boldsymbol{\eta}=\left[\begin{array}{c}
\boldsymbol{z}^{(k, r)} \\
\boldsymbol{z}^{(k, r-1)} \\
\vdots \\
\boldsymbol{z}^{(k, r-W+1)}
\end{array}\right], \boldsymbol{X}=\left[\begin{array}{c}
\left(\boldsymbol{x}^{(k, r)}\right)^{\mathrm{T}} \otimes \boldsymbol{I}_{M} \\
\left(\boldsymbol{x}^{(k, r-1)}\right)^{\mathrm{T}} \otimes \boldsymbol{I}_{M} \\
\vdots \\
\left(\boldsymbol{x}^{(k, r-W+1)}\right)^{\mathrm{T}} \otimes \boldsymbol{I}_{M}
\end{array}\right]
$$

where $\otimes$ is the Kronecker product and $\boldsymbol{I}_{M}$ is the identity matrix of size $M$.

Let $\boldsymbol{h} \in \mathbb{C}^{M^{2}}$ be a vector obtained by stacking the columns of $\boldsymbol{H}^{(k)}$. The last $W$ received symbol vectors can then be described as

$$
\gamma=\left[\begin{array}{c}
\boldsymbol{y}^{(k, r)} \\
\boldsymbol{y}^{(k, r-1)} \\
\vdots \\
\boldsymbol{y}^{(k, r-W+1)}
\end{array}\right]=\boldsymbol{X} \boldsymbol{h}+\boldsymbol{\eta}
$$

Taking advantage of (5), one can obtain an estimate of $\boldsymbol{h}$ by using a Moore-Penrose pseudoinverse

$$
\hat{\boldsymbol{h}}=\left(\boldsymbol{X}^{\mathrm{H}} \boldsymbol{X}\right)^{-1} \boldsymbol{X}^{\mathrm{H}} \gamma .
$$

Re-stacking the elements of $\hat{\boldsymbol{h}}$ column-wise leads to the desired channel matrix estimate $\hat{\boldsymbol{H}}^{(k)}$.

\section{B. RRH channel estimator}

An alternative approach is to estimate a single row of the copper channel matrix $\boldsymbol{H}^{(k)}$ at each RRH, feeding back the estimation results instead of the received symbols. This estimation procedure relies on the fact that transmit pilot symbol vectors $\boldsymbol{x}^{(k)}$ can be reconstructed at each receiver as long as they are aware of the $N_{I D}^{\text {cell }}$ values used in the system.

Let $W$ represent the number of symbols in an observation window such that $W \geq M$. Rewrite (2) as

$$
y_{i}^{(k)}=\underline{\boldsymbol{h}} \boldsymbol{x}^{(k)}+z_{i}^{(k)},
$$

where $\underline{\boldsymbol{h}}=\left[H_{i, 1}^{(k)}, \ldots, H_{i, i}^{(k)}, \ldots, H_{i, M}^{(k)}\right]$ is the vector corresponding to the $i^{\text {th }}$ row of channel matrix $\boldsymbol{H}^{(k)}$. Next, define $\underline{\boldsymbol{X}} \in \mathbb{C}^{W \times M}$ and $\underline{\boldsymbol{\eta}} \in \mathbb{C}^{W}$ as

$$
\underline{\boldsymbol{X}}=\left[\begin{array}{c}
\left(\boldsymbol{x}^{(k, r)}\right)^{\mathrm{T}} \\
\left(\boldsymbol{x}^{(k, r-1)}\right)^{\mathrm{T}} \\
\vdots \\
\left(\boldsymbol{x}^{(k, r-W+1)}\right)^{\mathrm{T}}
\end{array}\right], \underline{\boldsymbol{\eta}}=\left[\begin{array}{c}
z_{i}^{(k, r)} \\
z_{i}^{(k, r-1)} \\
\vdots \\
z_{i}^{(k, r-W+1)}
\end{array}\right] .
$$

The last $W$ symbols received by RRH $i$ can be written as

$$
\underline{\gamma}=\left[\begin{array}{c}
y_{i}^{(k, r)} \\
y_{i}^{(k, r-1)} \\
\vdots \\
y_{i}^{(k, r-W+1)}
\end{array}\right]=\underline{\boldsymbol{X}}^{\mathrm{T}}+\underline{\boldsymbol{\eta}} .
$$

The Moore-Penrose pseudoinverse then leads to the estimate

$$
\underline{\hat{\boldsymbol{h}}}^{\mathrm{T}}=\left(\underline{\boldsymbol{X}}^{\mathrm{H}} \underline{\boldsymbol{X}}\right)^{-1} \underline{\boldsymbol{X}}^{\mathrm{H}} \underline{\boldsymbol{\gamma}} .
$$




\section{Crosstalk Mitigation Performance}

In order to assess the performance of the proposed RRU architecture and channel estimation methods we have executed time domain simulations. Initially, the Vienna LTE link level simulator [11] was used to generate downlink LTE radio frames. Each cell was configured to use a single antenna port, and its $N_{I D}^{\text {cell }}$ number was assigned according to (4).

We have measured direct and FEXT transfer functions for six pairs in telephony-grade copper cables [12] with different lengths. The frequency range of interest is from 21 to $24 \mathrm{MHz}$, in accordance to the system proposed in [3], [4].

The simulations were divided in two stages. At first, for each pilot subcarrier, a channel matrix estimate $\hat{\boldsymbol{H}}$ is obtained using the methods proposed in the previous section. For subcarriers not loaded with pilot symbols, channel estimates were obtained via linear interpolation. Next, each estimated channel matrix $\hat{\boldsymbol{H}}$ was used to calculate a diagonalizing precoder $\boldsymbol{P}=(1 / u) \hat{\boldsymbol{H}}^{-1} \operatorname{diag}\left\{\hat{H}_{1,1}, \ldots, \hat{H}_{M, M}\right\}$, where $\operatorname{diag}\left\{\hat{H}_{1,1}, \ldots, \hat{H}_{M, M}\right\}$ represents a diagonal matrix with elements $\hat{H}_{1,1}, \ldots, \hat{H}_{M, M}$ along its main diagonal. The normalization factor $u$ is chosen to guarantee that the precoding operation does not lead to increases in transmit power [6]. These precoders were then used in simulations against the measured channels $\boldsymbol{H}$.

To quantify performance we calculate EVM as specified in [13]. 3GPP specifies limit EVM values for each of the constellations used for LTE's physical downlink shared channel (PDSCH). The limits are $17.5 \%, 12.5 \%, 8 \%$ and $3.5 \%$ for QPSK, 16-QAM, 64-QAM and 256-QAM respectively.

For each loop length we have varied the additive noise power spectrum density (PSD), calculating EVM before the $\mathrm{RF}$ front-end at the RRH. The simulation results for the worst performing cell are gathered in Fig. 4.

The performance for a baseline system, deployed over a 100 meters of cable without crosstalk cancellation, is represented in Fig. 4 by a dashed blue line with pentagram markers. The resulting EVM would limit the system to using QPSK modulation. For longer cables without crosstalk cancellation, the EVM curves exceed the scale of the plot and 3GPP compliance is infeasible.

As a reference, single user bounds are depicted as dotted lines for all cable lengths. These represent the EVM when the cell in question is the only active transmitter.

The solid lines represent the performance with diagonalizing precoders and approach the single user bounds for all cable lengths. The channel estimates used to design the precoders were obtained using the feedback-based estimator (Sec. III-A) with $W=20$, corresponding to the number of pilot symbols observed in one LTE frame. Results for the RRH estimator (Sec. III-B) were equivalent and therefore omitted.

The gap between EVM limits (red dotted lines) and a certain curve indicate the margin left for implementation losses. As an example, consider that one would design an LTE-over-copper system for deployment over 300 meters of copper, subject to a background noise level of $-140 \mathrm{dBm} / \mathrm{Hz}$. In order to support 256-QAM implementation losses caused, for example, by imperfections in the RF front-end would be limited to $3.5 \%$.

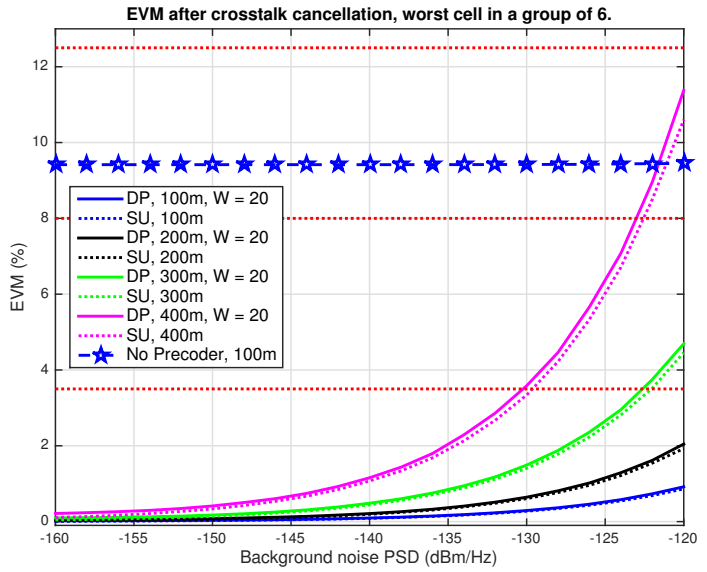

Fig. 4. EVM performance for the worst cell in a group of six LTE-overcopper systems, deployed over different cable lengths. DP represents the curves obtained with a diagonalizing precoder, while SU represents single user bounds.

\section{CONCLUSION}

We have presented an architecture for crosstalk mitigation in LTE-over-copper systems. It is transparent to baseband processors and can be implemented in a radio unit, where up and down-conversion are performed. Two channel estimation methods that take advantage of LTE's downlink reference signals were also presented.

\section{REFERENCES}

[1] J. Gambini and U. Spagnolini, "Wireless over cable for femtocell systems," IEEE Communications Magazine, vol. 51, no. 5, pp. 178-185, May 2013.

[2] C. Lu, M. Berg, E. Trojer, P.-E. Eriksson, K. Laraqui, O. V. Tidblad, and H. Almeida, "Connecting the dots: small cells shape up for highperformance indoor radio," Ericsson Review, vol. 91, December 2014.

[3] Y. Huang, E. Medeiros, S. Höst, T. Magesacher, P.-E. Eriksson, C. Lu, P. Ödling, and P. O. Börjesson, "Enabling DSL and Radio on the Same Copper Pair," in Proc. IEEE International Conference on Communications (ICC), 2015

[4] - "LTE Over Copper - Potential and Limitations," in Proc. 2015 IEEE 26st International Symposium on Personal Indoor and Mobile Radio Communications (PIMRC), 2015.

[5] G. Ginis and J. Cioffi, "Vectored transmission for digital subscriber line systems," IEEE Journal on Selected Areas in Communications, vol. 20, no. 5, pp. 1085-1104, 2002.

[6] R. Cendrillon, G. Ginis, E. Van den Bogaert, and M. Moonen, "A near-optimal linear crosstalk precoder for downstream VDSL," IEEE Transactions on Communications, vol. 55, no. 5, pp. 860-863, 2007.

[7] Self-FEXT Cancellation (Vectoring) for Use with VDSL2 Transceivers, ITU Recommendation, Sep. 2010.

[8] Fast Access to Subscriber Terminals (G.fast) - Physical Layer Specification, ITU Recommendation, Dec. 2014.

[9] 3GPP, "LTE; Evolved Universal Terrestrial Radio Access (E-UTRA); Physical channels and modulation," 3rd Generation Partnership Project (3GPP), TS 36.211 V12.7.0, October 2015.

[10] Alcatel-Lucent Bell, "G.vdsl: On the tracking speed required for changing crosstalk channels," ITU-T SG15 Contribution, May 2007. [Online]. Available: https://www.itu.int/md/T05-SG15-C-0540

[11] S. Schwarz, J. Ikuno, M. Simko, M. Taranetz, Q. Wang, and M. Rupp, "Pushing the limits of LTE: A survey on research enhancing the standard," IEEE Access, vol. 1, pp. 51-62, 2013.

[12] Ericsson AB, Access Network Pair cable, TEL 312, 2010. [Online]. Available: http://goo.gl/4RdCXc

[13] 3GPP, "Evolved Universal Terrestrial Radio Access (E-UTRA); Base Station (BS) radio transmission and reception," 3rd Generation Partnership Project (3GPP), TS 36.104 V12.8.0, July 2015. 